\title{
Wine Science in the Omics Era: The Impact of Systems Biology on the Future of Wine Research
}

\author{
D. Rossouw and F. F. Bauer* \\ Institute for Wine Biotechnology, University of Stellenbosch, Stellenbosch, South Africa \\ Submitted for publication: May 2009 \\ Accepted for publication: July 2009 \\ Key words: Systems biology, wine, yeast, transcriptomics, proteomics, metabolomics
}

\begin{abstract}
Industrial wine making confronts viticulturalists, wine makers, process engineers and scientists alike with a bewildering array of independent and semi-independent parameters that can in many cases only be optimized by trial and error. Furthermore, as most parameters are outside of individual control, predictability and consistency of the end product remain difficult to achieve. The traditional wine sciences of viticulture and oenology have been accumulating data sets and generating knowledge and know-how that has resulted in a significant optimization of the vine growing and wine making processes. However, much of these processes remain based on empirical and even anecdotal evidence, and only a small part of all the interactions and cause-effect relationships between individual input and output parameters is scientifically well understood. Indeed, the complexity of the process has prevented a deeper understanding of such interactions and causal relationships. New technologies and methods in the biological and chemical sciences, combined with improved tools of multivariate data analysis, open new opportunities to assess the entire vine growing and wine making process from a more holistic perspective. This review outlines the current efforts to use the tools of systems biology in particular to better understand complex industrial processes such as wine making.
\end{abstract}

Grapevine growing and wine making have been a part of human agricultural activity for thousands of years. Today, grapes are the most planted fruit crop in the world, and the global wine industry has become a multi-billion dollar business. Yet, the process of vine growing and wine making continues to present tremendous challenges. The traditional wine sciences of viticulture and oenology are challenged by the complexity of the process, and many studies, while reporting on the effects of individual parameters, all too frequently fail to establish causality. The input variables in the vineyard involve a large number of factors that influence the growth of the grapevine and the composition of the grape berries, and in particular include many environmental factors such as soil, aspect, slope, and climate. These factors interact with and impact on the genetic potential of individual grapevine cultivars or rather individual plants. From a wine making perspective, the relevant end result of these processes is defined by the chemical composition of the grape, otherwise known as the grape metabolome (Driesel et al., 2003; Da Silva et al., 2005; Cramer et al., 2007). Traditional research on grapevine biology (physiological, genetic and molecular approaches) has helped to establish broad correlations between specific environmental factors and aspects of the final grape and must composition. On the whole though, our current understanding of grapevine biology is curtailed by an incomplete molecular map and limited knowledge regarding the genetic regulation of this complex woody perennial.

On the oenological side many factors will contribute to transform and give expression to the grape metabolome, and will impact on the character and quality of the final product. Such factors include the treatment of the grapes and of the must before fermentation, the physical parameters prevalent during fermentation, and the impact thereof on dynamic microbial ecosystem that will continuously adapt and change while alcoholic fermentation proceeds. This wine fermentation ecosystem usually includes numerous strains of lactic and acetic acid bacteria, as well as a large spectrum of yeast species and other fungi. From an oenological perspective, the most relevant of these organisms is the scientifically well studied yeast Saccharomyces cerevisiae. This yeast appears best adapted to the harsh environmental conditions prevalent during wine fermentation, such as high osmotic pressure, low $\mathrm{pH}$ and, in particular, the increasing levels of ethanol (Attfield, 1997). As a consequence, commercial wine yeast strains indeed are almost exclusively of this species, and spontaneously fermenting musts also usually end up with one or more $S$. cerevisiae strains as the dominant yeast (Frezier \& Dubourdieu, 1992). For this reason most research on alcoholic fermentation has centered on this organism.

However, our current knowledge of grapevine and microbial biology and of the chemical processes that result in a specific wine remains limited. This lack of knowledge and understanding significantly limits our ability to improve wine quality and consistency. In particular, modern biotechnological approaches are knowledge-based, and we are only able to change or manipulate a biological system to the extent that we understand its functioning. While the amount of data describing biological systems has been increasing rapidly, this increase has been largely built on approaches that can be qualified as reductionism. Such approaches focus on individual components, such as a single gene or protein within a biological system, and have contributed tremendously to our understanding of biological systems, in particular by mapping genetic and metabolic pathways and fluxes. However, they are inherently incapable of elucidating the

"Corresponding author: e-mail: fb2@sun.ac.za

Acknowledgements: Funding for ongoing research in this field is provided by the NRF and Winetech, and personal sponsorship by the Wilhelm Frank Trust. 
nature of the complex biological networks that characterize living organisms. Ultimately, complex systems can only be interpreted by complex, high-level analyses.

With the recent development of new technologies in the biological and chemical sciences, as well as improvements in statistical and interpretation tools, such high level analyses have become a feasible option, and a unique opportunity exists to approach the analysis of biological systems in a holistic manner. These approaches have been loosely integrated under the term "systems biology" which is referring to the large number of novel approach to investigate biological processes on a whole cell or whole organism level.

Conveniently, $S$. cerevisiae also happens to be the traditional model organism of choice for molecular and cellular biologists. For this reason, $S$. cerevisiae will be the main focus of the following sections in this paper and will serve to highlight the role of 'omic'-applications in wine science and research.

\section{Yeast biotechnology in the food and beverage industry}

Indigenous fermented foods such as bread, cheese and wine have been prepared and consumed for thousands of years, and it is estimated that fermented foods contribute to about one-third of the diet worldwide. Biotechnology in the food and beverage sector targets the selection and improvement of yeast strains with the objectives of improving process control, yields and efficiency as well as the quality, safety and consistency of the end-product (Chapman, 1991).

Wine and beer represent the two most popular products of alcoholic fermentation processes. The commercial yeast strains that are used in these processes have been primarily selected for their fermentation efficiency. However, besides the conversion of sugars to alcohol and $\mathrm{CO}_{2}$, yeast metabolism results in the production of a diversity of metabolites, including vitamins, antimicrobial compounds, amino acids, organic acids (e.g. citric acid, lactic acid) and flavour compounds (e.g. esters and aldehydes). These metabolites make an important contribution to the character and quality of the final product, in particular with regard to aroma, flavour, and microbiological stability (Lambrechts \& Pretorius, 2000). A considerable volume of current research both in academia and industry therefore targets the application of yeast biotechnology to improve fermentation efficiency and the production, quality and yields of metabolites (Cereghino \& Cregg, 1999; Stephanopoulos et al., 2004).

Traditional methods of genetic improvement such as classical mutagenesis and hybridization have been used in the improvement of yeast strains which are widely used industrially in baking, brewing and wine making (Pretorius \& Bauer, 2002). Recombinant DNA approaches have also been used for genetic modification of yeast strains to promote the expression of desirable genes, to hinder the expression of others, to alter specific genes or to inactivate genes so as to block specific pathways. In the field of wine science specifically, genetic modification of wine yeast for improved secretion of oenologically relevant enzymes (Malherbe et al., 2003; Louw et al., 2006), production of aroma compounds (Lilly et al., 2006 a,b), glycerol production (Cambon et al., 2006), malate degradation (Volschenk et al., 1997 a,b) and decreased ethanol production (Heux et al., 2006) has proven to be a feasible endeavour.
Several genetically modified yeasts appropriate for brewing, baking and wine making have been approved for use, although, as far as can be ascertained, none of these strains have been widely used commercially in the past. The possibilities for further engineering improved yeast strains are however clearly enormous.

\section{Systems biology background}

Metabolic engineering is the rational alteration of the genetic architecture of an organism to achieve a specific phenotype (Bailey, 1991). Classic 'bottleneck engineering' targeting the socalled rate-limiting steps in a pathway has only met with partial success. This is because cells are comprised of a complex network of regulatory mechanisms that counteract genetic modifications such as those derived from mutations by employing alternative pathways for continued robust performance (Farmer \& Liao, 2000). Control of metabolic processes is in part hierarchical, with information transfer occurring from the genome to the transcriptional level, moving on to translation and finally enzyme activity. However, feed-back loops among the different levels are numerous. 'Omics' technologies today can analyze and monitor entire classes of biological macromolecules, such as DNA, RNA and proteins, as well as metabolites on a whole cell, whole tissue, whole organism or whole population level (Brown \& Botstein, 1999; Bruggeman \& Westerhoff, 2007). Such omics-based technologies have led to the establishment of fields of expertise referred to as transcriptomics, proteomics and metabolomics, depending on the specific layer of biological information that is being monitored. Ideally, in a systems analysis approach, all biochemical components that are involved in the process of interest should be monitored. While most of these analyses have thus far been focusing on quantification, other technologies aim to determine the interactions between components (interactomics) and the genetic or metabolic flux (fluxomics) within the system.

Taken together, such data can allow the reconstruction of in silico biological networks (Goryanin et al., 1999). The properties of the reconstructed network are in principle amenable to mathematical modeling, allowing incorporation into computer models that can be interrogated systematically to predict biological functions and system responses to specific perturbations (Palsson, 2000; Price et al., 2003).

The large volumes of data generated by these approaches necessitates concomitant development in fields known as bioinformatics and multivariate data analysis (Palsson, 2002; Ge et al., 2003; Larsson et al., 2006; Lavine \& Workman, 2006). Fortunately for the wine sciences, $S$. cerevisiae retains its title as one of the preferred model organism in the field of systems biology and bioinformatics as well. This has meant that many cutting edge 'omics' technologies and supporting statistical analysis modules are routinely available for research on wine yeast strains, as will be discussed in the following sections.

\section{1-Genomics}

The general starting point of any system-wide analysis is usually at the genome level, as phenotypic features and changes therein are due to changes in the primary genome sequence of a particular organism. Whole genome sequencing is the process whereby the complete DNA sequence of an organism's genome is determined at a single time. This entails sequencing all of an organism's 


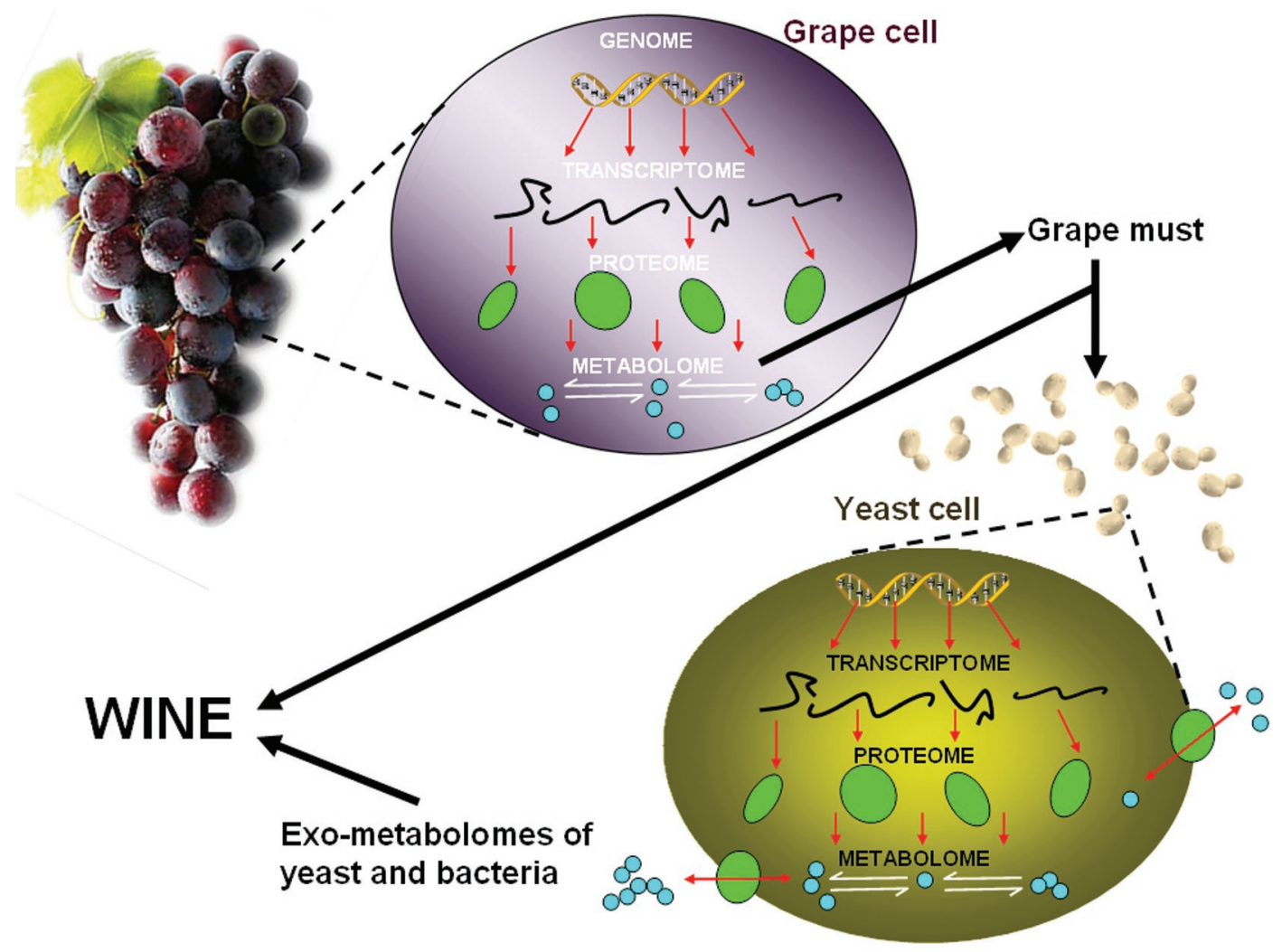

FIGURE 1

Representation of the different biological information layers that impact on the wine making process from grape to must to wine. The grape metabolome is the result of the interaction of the various layers of the biological information transfer system and will define the composition of the must. The grape must is transformed through the action of various microorganisms to obtain the final product, wine. This biological system represents the entire wine making process and can be holistically investigated through the use of omics tools that monitor the totality of - or as many as possible - components representative of each layer.

chromosomal DNA as well as DNA contained in the mitochondria. Whole genome sequencing has changed in the most profound way the manner in which scientists plan and perform research as gene sequences have provided enabling information and resources for a wide variety of scientific applications.

$S$. cerevisiae was one of the first organisms to have its genome completely sequenced, more than 10 years ago (Goffeau et al., 1996). This breakthrough in yeast research opened the door for yeast biologists to gain insight into yeast physiology on a molecular level. One of the main goals of genome sequencing is to identify all the genes in an organism: Computational methods for protein-coding gene identification are reasonably well developed, especially for compact genomes such as that of $S$. cerevisiae, which has a coding density of around 75\% (Goffeau et al., 1996). The genome of the original S288c laboratory strain is thus well annotated, with clearly delineated coding regions and regulatory elements, and is easily accessible to interested researchers.

In the case of wine yeast strains, however, increased complexity becomes an important factor: These yeasts exhibit great variation in chromosome size and number in comparison to laboratory strains, and are also aneuploid (Bakalinsky \& Snow, 1990). Chromosomal changes include gain or loss of whole chromosomes and large-scale deletions and/or duplications (Adams et al., 1992; Rachidi et al., 1999). Unfortunately very few DNA sequences of wine yeasts have been published or are publicly accessible in databases (Masneuf et al., 1998). Overall though, the sequence homology between the laboratory strain S288c and wine yeasts is approximated at around
99\% (Masneuf et al., 1998), which means that sequence information from the S288c strain can be used for general systematic analysis of wine yeast strains (Puig et al., 1998, 2000).

Recently a major milestone in wine yeast genomics was reached when the Australian Wine Research Institute completed the genome sequencing of the commercial yeast AWRI1631 (Borneman et al., 2008). Interestingly, about $0.6 \%$ of this sequence information differed from that of the laboratory strain S288c, and extra DNA sequences (enough to carry at least 27 genes) were discovered in the wine yeast. Most of the sequences of these putative genes do not resemble anything found in other species of Saccharomyces, but appear to be similar to genes found in distant fungal relatives. Some of these wine yeast-specific genes seem to encode proteins that are associated with the cell wall, and could thus account for the ability of wine yeasts to survive the harsh prevailing conditions in winemaking environments. Other functionalities include genes coding for proteins involved in amino acid uptake, which potentially holds implications for wine aroma and flavour development due to the association of amino acid metabolism with volatile aroma compound production.

Three decades have passed since the invention of electrophoretic methods for DNA sequencing, and advancements in the efficiency and cost-effectiveness of sequencing has made rapid sequencing of small genomes financially and practically feasible. Various novel sequencing technologies are being developed, as well as software tools for automated genome annotation, together aspiring to reduce costs and time frames for genome analysis. This means that many 
more wine yeast genomes will be sequenced and become publicly available in the near future. Comparative genomics will thus become a major tool for the insightful interpretation of genomic data within the wine-making context.

\section{2-Transcriptomics}

As mentioned, system-wide endeavours tend to start at the genomic level, since phenotypic changes are due to perturbations of gene sequence and transcriptional levels. In the decade following the sequencing of the $S$. cerevisiae genome a whole suite of analysis tools were developed based on gene sequence knowledge and functional annotation of $90 \%$ of the coding sequences in the yeast genome. The challenge of large-scale functional genomics followed as the next key step in the pursuit of complete understanding of yeast physiology and metabolism. Functional genomics, a relatively new area of research, aims to determine patterns of gene expression and interaction in the genome. It can provide an understanding of how yeast responds to environmental influences at the genetic level, and should therefore allow adaptation of conditions to improve technological processes. As previously mentioned, there is considerable variation in the fermentation performance of different wine yeast strains in terms of the secretion of oenologically relevant enzymes, the tendency to result in 'stuck' or 'sluggish' fermentations, the production of aroma compounds and the production of off-flavours. In short, the strains differ drastically in terms of the quality of wine that they produce. These variations across strains of $S$. cerevisiae are inheritable, and thus genetically determined. Functional genomics holds the potential to shed light on genetic differences allowing some strains to perform better than others with regard to certain desirable processes. It also holds great promise for defining and modifying elusive metabolic mechanisms used by yeast to adapt to different environmental conditions.

The technology of transcriptomics is a result of the convergence of several technologies, such as DNA sequencing and amplification, synthesis of oligonucleotides, fluorescence biochemistry, and computational statistics. It basically confers the ability to measure mRNA abundance (Lander, 1999), which reveals the effects of the global physiological and metabolic control machinery on transcription by identifying differentially expressed genes. It is thus possible to observe the expression of many, if not all genes simultaneously, including those with unknown biological functions, as they are switched on and off during normal growth, or while the yeast attempts to cope with ever-changing environmental conditions such as those encountered during fermentation. By identifying similarities in the transcriptional profile, the role of many previously uncharacterized genes was predicted, based on the assumption that coexpressed genes are functionally related. An early example of such studies was the identification of genes that were differentially expressed in Saccharomyces cerevisiae in response to a metabolic shift from growth on glucose to diauxic growth on glucose and ethanol (DeRisi et al., 1997).

Numerous yeast transcriptomics studies have also been conducted in chemostat cultures, which revealed, among others, that growth-limiting nutrients have a profound impact on genomewide transcriptional responses of yeast to process perturbations and/ or molecular genetic interventions (Boer et al., 2003; Usaite et al., 2006). Transcriptomic profiling of yeast exposed to various stress conditions has likewise provided insights into the effects of those stresses on the cell at the transcriptional level (Gasch et al., 2000; Kuhn et al., 2001; Gasch \& Werner-Washburne, 2002). These examples of iterative perturbations and systematic phenotype characterization (on a gene expression level) have yielded a plethora of system insights that have revolutionized microbial biology.

Several transcriptomic studies have also been published for research conducted with wine yeast strains (Erasmus et al., 2003; Rossignol et al., 2003; Varela et al., 2005; Mendes-Ferreira et al., 2007; Marks et al., 2008; Pizarro et al., 2008; Rossouw \& Bauer, 2008). These studies have illuminated the intrinsic genetic and regulatory mechanisms involved in fermentation, and have greatly increased our understanding of this important process. For instance, Pizarro et al. (2008) showed how growth temperature and nitrogen limitation affects differential physiological and transcriptional responses in both laboratory and wine strains of $S$. cerevisiae. Nitrogen metabolism was found to be deregulated in the wine yeast (allowing simultaneous uptake of different nitrogen sources), which explains the competitive advantage of wine yeast strains in real wine fermentations. This accounted for the ability of wine yeast cells to proliferate and produce comparatively higher biomass yields at low temperatures with the same amount of nitrogen compared to laboratory yeasts.

\section{3-Proteomics}

Moving from the gene to the protein level brings us to proteomics, an approach aiming to identify and characterize complete sets of proteins, and protein-protein interactions in a given species (Hartwell et al., 1999; Ideker et al., 2001). An increased transcript level cannot be interpreted as evidence for a contribution of the encoded protein to the cellular response in the immediate experimental context. But even though gene expression might not relate directly to protein expression (Ideker et al., 2001), the protein products of genes that are coexpressed under different conditions are often functionally related with one another as part of the same pathway or complex (Grigoriev, 2001; Ge et al., 2001). Considering, however, that transcript levels are not directly correlated to protein levels and in vivo fluxes (Griffin et al., 2002; Washburn et al., 2003; Daran-Lapujade et al., 2004), large-scale transcriptomic datasets need to be combined with other data subsets such that the overlapping set of interactions provides more insightful and meaningful information on the system in question (Tong et al., 2002). Combining many layers of systematic cell and molecular biology such as protein levels and transcript expression data enables the construction of an accurate information matrix and a complete cellular map (Walhout et al., 2002).

Genome-scale protein quantification is not yet feasible, but methods for determining relative levels of protein between samples have been developed (Smolka et al., 2002). Conventional quantitative proteome analysis utilizes two-dimensional (2D) gel electrophoresis (O'Farrell, 1975) to separate complex protein mixtures followed by in-gel tryptic digestion and mass spectrometry for the identification of protein. More than 1500 soluble proteins of yeast are detectable and well separated of two-dimensional gels. This technique offers the opportunity to detect alterations in protein synthesis, protein modifications, and protein degradation occurring in response to environmental or genetic changes. However, the twodimensional gel approach suffers from the low number of proteins which are identified on the yeast protein map, as well as poor gel- 
to-gel reproducibility, the under-representation of low-abundant and hydrophobic proteins and the poor dynamic range of detection (Fey \& Larsen, 2001; Rabilloud, 2002).

To overcome some of these limitations, high-throughput chromatography in combination with mass spectrometry can be used for fast and accurate protein identification, as long as the protein/s already exist/s uniquely in a sequence database (Mann et al., 2001). The most commonly used high-performance liquid chromatographic (HPLC) approach for the separation of peptides from protein digests in complex proteomic applications is $2 \mathrm{D}$ nano-liquid chromatography-mass spectrometry (LC/MS). In this approach, a strong cation exchange (SCX) column is used for the first dimension and a reversed phase (RP) column for the second (Nägele et al., 2004). A total of 1504 yeast proteins have been unambiguously identified in a single analysis using this $2 \mathrm{D}$ chromatography approach coupled with tandem mass spectrometry (MS/MS) (Peng et al., 2002).

In fermenting yeast, the first forays into proteomics have been reported, usually in conjunction with transcriptomic ormetabolomic analysis (Brejning et al., 2005; Salvadó et al., 2008). Such studies have increased our knowledge regarding the growth phases of fermenting yeasts, and have suggested new methodologies for optimization and control of growth during fermentation-based industrial applications. Proteome studies of yeast responses to various stress conditions have also increased our knowledge of the functional modules involved in yeast responses to specific environmental factors (Vido et al., 2001; Kolkman et al., 2006). For example, Kolkman et al. (2006) compared yeast responses to nitrogen and carbon limitation: Interestingly, comparison of transcript and protein levels clearly showed that increases in protein levels in response to glucose limitation was mainly transcriptionally controlled, whereas upregulation of proteins in response to nitrogen limitation was essentially controlled at the posttranscriptional level by increased translational efficiency and/ or decreased protein degradation.

Another important goal of functional proteomics is the identification of functional modules based on the knowledge of protein action. Protein-protein interactions play a crucial role in elucidating the nature of these mechanisms. Innovative methods for the cell-wide analysis of protein interactions and signaling pathways have been developed in recent times (Templin et al., 2004). These include the high-throughput yeast two-hybrid systems (Uetz et al., 2000; Ito et al., 2001), protein arrays (Walter et al., 2000; Zhu \& Snyder, 2003; Weiner et al., 2004), and fluorescence-based interaction assays (Hu \& Kerppola, 2003). In contrast to clustering genes, clustering protein interactions reveals modules which have similar functionalities and are therefore more closely associated in bringing about a particular response. For yeast specifically, the protein interactions from a wide range of experiments were transformed into a weighted network, with the weights representing the experimentally determined confidence levels for a particular interaction (Pereira-Leal et al., 2004). Such models of proteinprotein interactions in yeast form an invaluable framework for future analysis and evaluation of 'omic' data-types.

\section{4-Metabolomics}

Strain phenotype characterization has relied primarily on transcript abundance and protein measurements. Only rarely have small metabolites been included in the analysis of the system due to difficulties in sampling and analyzing these molecules. The major complication is the rapid time scales of change, or oscillations in the levels of metabolites in a pathway, even if this pathway is in a balanced, unperturbed state of equilibrium. Small molecules also cover a wider range of chemical characteristics than do RNA transcripts, for example, and are more difficult to measure simultaneously (Dettmer et al., 2006).

Despite all the above-mentioned complications, advances in high-throughput methodologies in analytical chemistry now allow the detection and relative quantification of a large number of metabolites simultaneously (Dunn \& Ellis 2005; Smedsgaard \& Nielsen 2005; Villas Bôas et al., 2005). Gas chromatography coupled to mass spectrometry allows high-throughput analysis in a relatively short time and at a fairly low cost. The gas chromatograph separates metabolites while the mass spectrometer identifies and quantifies metabolites corresponding to a given standard peak. Specifically, the chemical analysis of wine has made tremendous progress over the last decade, and it is now possible to quantify a large number of chemical compounds (both volatile and non-volatile) with relative accuracy (Villas Bôas et al., 2005). In addition to gas or liquid chromatography -mass spectrometry (GCMS or LCMS), the development of two dimensional techniques has increased throughput and effectiveness by allowing for the analysis of compounds with different physiochemical properties in one analysis (Adahchour et al., 2006; Campo et al., 2006).

Metabolites are known to be involved as key regulators of systems homeostasis. As such, level changes of specific groups of metabolites may be descriptive of systems responses to environmental interventions. Their study is therefore a powerful approach for characterizing complex phenotypes, as well as for identifying biomarkers for specific physiological responses. Globally assaying metabolic states does present the opportunity to identify a more diverse set of active molecular relationships, particularly in the context of high-level regulation of transcriptional and translational processes by certain metabolites. Metabolic profiles can be used to define a 'footprint' of processes that occur in response to developmental, genetic or environmental effects, and are thus useful in defining the cellular phenotype (Allen et al., 2003). Metabolic data can also be incorporated into databases that integrate transcription, protein-protein interactions and metabolism to identify multilevel sub-networks which are activated in response to a given perturbation to the system.

To complement these types of analysis, chemometrics approaches have evolved to enable these large data sets to be mined for information through multivariate analysis of multi-level datasets. The broader metabolic state characterization of fermenting yeast should allow better understanding of the interplay between different pathways and may enhance our ability to identify key cellular mechanisms (Çakir et al., 2006). Metabolomics studies focused on yeast have been on the increase over the past few years (Daran-Lapujade et al., 2004; Beltran et al., 2006; Kresnowati et al., 2006; Villas-Bôas et al., 2007), and have provided new insights into molecular events associated with the responses of yeast to environmental factors such as fermentation temperature and the availability of carbon substrates. For example, Kresnowati et al. (2006) showed that upon relief of glucose limitation cellular homeostasis is restored by a significant increase in trehalose-6P 
levels, followed by the coordinated downregulation of the three hexokinase encoding genes as part of a response to prevent 'glucose-accelerated death'. Immediate adjustment of fructose2,6P levels was also evident, thus providing evidence for the cellular mechanisms responsible for adjusting metabolite levels in central carbon metabolism.

\section{5-Fluxomics}

Another noteworthy obstacle to the rational optimization of yeast for industrial purposes is the lack of a reliable, global metabolic model that captures the majority of the stoichiometric, kinetic and regulatory effects on metabolite interconversions and metabolic flux distribution through the cellular reaction network (Edwards \& Palsson, 2000). In order to gain full understanding of the metabolism of any cell/tissue/ organism, both levels of metabolites and their fluxes need to be studied. Flux determination is thus an essential component of strain evaluation for metabolic engineering (Stephanopoulos, 1999). New methods for flux measurement have been developed based on NMR and MS technologies which allow accurate, high resolution measurements of pathway flux to be made using radiolabeled substrates (Szyperski, 1998; Christensen $\&$ Nielsen, 1999). Such methods are based on feeding ${ }^{13} \mathrm{C}$-labeled substrates to the cell for the characterization of metabolism (Wiechert, 2001). For example, feeding of cells with ${ }^{13} \mathrm{C}$-labeled glucose followed by analysis of the ${ }^{13} \mathrm{C}$ enrichment pattern in different intracellular metabolites establishes an experimental platform for the calculation of real flux estimates (Gombert et al., 2001). The tight connection of the different parts of metabolism means that changes in fluxes in one part of the metabolic network disseminate to many other related parts (Nielsen, 2003). Thus, with the development of the necessary mathematical frameworks, measurement of even a few metabolic fluxes may provide valuable information regarding complete metabolic networks (Wiechert et al., 1997).

Metabolic fluxes can be used to characterize phenotypes and carbon flow in a system due to any given perturbation (Nielsen, 2003) and forms an integral part of applications in yeast systems biology and metabolic engineering. Metabolic flux modeling can also be used to capture the genome-scale systems properties of an organism's metabolism and further facilitate the construction, validation, and predictive capabilities of models constructed from 'omic' information (Sauer, 2004). For instance, constraint-based network models have been developed using reaction stoicheometry to represent the biological network (Reed \& Palsson, 2003). Such models are built by connecting the metabolites and the reactions they participate in to form a metabolic graph which is then applied to optimize the reaction rates (fluxes) given a specific target function (i.e. biomass or fermentation product formation). These models have also been invaluable in predicting the impact of gene deletions in several model organisms (Edwards et al., 2001; Famili et al., 2003; Forster et al., 2003). Conveniently, genomescale models have also been constructed for yeast (Forster et al., 2003; Duarte et al., 2004), which means that predictions of carbon flux distribution can be integrated with other 'omics' data types for interpretation purposes and to further guide metabolic engineering strategies.

\section{6-Interactomics}

Ultimately, systems biology is faced with the formidable task of interpreting and contextualizing the diverse sets of biological data from the various levels of 'omic' analysis with the aim to elucidate the mechanisms behind complex biological phenomena. Comparisons of yeast transcriptomes and proteomes under different cultivation conditions have shown that multilevel analysis is essential for yeast systems biology (Kolkman et al., 2006) to avoid possibly missing the cause and effect relationships from other stages which impact the system as a whole.

The significant advances in genome sequencing, transcription, and protein and metabolite profiling have not always translated into successful metabolic engineering applications in yeast and other microbial systems. This is mainly due to a breach in the incorporation of these large datasets into meaningful models which explain how these components work in unison to produce the desired trait in the cell (Vemuri \& Aristidou, 2005). A comparative transcriptomic and exometabolomic analysis has been successful in predicting the impact of changes in expression levels of individual genes on the complex network of pathways that lead to the production of aroma compounds (Rossouw et al., 2008). The final component of a successful systems biology study is thus within the sphere of interactomics, which aims to integrate the transfer of information between the other phases of analysis with the use of mathematical modeling and simulation tools (De Jong, 2002).

Cellular computational models are becoming crucial for the analysis of complex biological systems. Various statistical methods, including pattern discovery and characterization tools, are available to create links between large data sets and phenotypes. Given enough data, it becomes possible to extract probabilistic models that can theoretically capture cellular interactions without prior knowledge of an interaction network (Jeong et al., 2000; De Jong et al., 2003). By building these cellular models, a comprehensive scaffold of molecular interactions is made available for mining to reveal a hierarchy of signaling, regulatory and metabolic pathways. Pathway maps can be extracted from this scaffold using computational models which identify the key components, interactions and influences required for more detailed interrogation using data from transcriptome or proteome analyses (Ideker \& Lauffenberger, 2003).

To summarize, the yeast cell is an elaborate network of molecular and environmental interactions that together bring about a highly complex phenotype. Understanding the functional consequences of the biomolecular interactions that occur in the yeast is a pre-requisite to understanding the relationship between yeast and must, and how each is changed by the other during the course of fermentation. Although we are still limited in our understanding of regulatory phenomena from a global perspective, high-throughput 'omic' techniques have the potential to provide such information. In particular, the combination of comparative microarray datasets with existing models of yeast metabolism and interaction networks offers the potential for in silico evaluation of biologically relevant gene expression changes in the context of key areas of metabolism (Förster et al., 2003; Patil \& Nielsen, 2005). This approach is one of several accessible multi-level analyses that can be applied to fermenting yeast, and holds great potential in terms of providing answers to scientific questions of fundamental importance.

\section{Systems biology meets biotechnology}

The emergence of systems biology constitutes a massive paradigm shift for biotechnologists. The switch from reductionist approaches 
in molecular biology to a new school of biological thought that is dominated by integrative 'big-picture' thinking opens new perspectives for the design and implementation of biotechnological approaches. The high throughput technologies of the post-genomic era have effectively created a massive amount of largely unexplored datasets, mostly publicly available in various databases. Systems biology may hold the key to assimilating all this information together into coherent models that facilitate drug discovery and metabolic engineering, the two hubs of modern biotechnology. For a more complete review of the impact of systems biology on metabolic engineering, see Nielsen \& Jewett (2008).

There is undoubtedly a desperate need for novel alternatives to the hit-and-miss approaches of bioprocess and bioproduct development in the past. A few systems biology companies have already emerged in the last decade, mostly in the spheres of drug discovery and development, as well as signal transduction. Global biotech companies such as Novartis have also embraced systems biology divisions into the folds of their existing corporate structure (Mack, 2004). By using systems biology approaches, established pharmaceutical companies have managed to drastically reduce their 'screening to compound development' periods in drug development for diabetes, obesity, arthritis, asthma, several cancers and many more money-spinning diseases. Outside of healthcare, the same opportunity for accelerated success exists for metabolic engineers as well.

Systems biology also has the potential to be a key role-player in the strain improvement arena of biotechnology (Stephanopoulos et al., 2004). Successful exploitation of cellular complexity for strain enhancement relies on a coordinated understanding of multiple cellular processes. Progress in this area is thus dependent on the development of theoretical frameworks that facilitate the elucidation of molecular mechanisms and the identification of genetic targets for modification. Strain improvement does present a very specific and attainable goal in the context of systems biology, particularly in combination with genetic tools such as the yeast overexpression and deletion libraries. Strains can be modified by introducing specific transport, conversion or regulatory changes that result in flux redistribution and improved production of desired compounds, or altered strain physiology. Clearly innovative application of relevant technologies holds the potential to expedite insightful modifications to yeast strains for application in the wine-making industry.

\section{CONCLUSION}

The idea of wine science as a convergence of multidisciplinary scientific exploits is a well known and established reality. Core sciences include biological, chemical, ecological, geological and sensory sciences, as well as certain aspects of process and chemical engineering. As understood by everyone in the field, the process of winemaking on a large-scale agricultural and industrial level involves numerous interlinked factors that are to a large extent poorly characterized and even more poorly controlled. While the more classic methods of scientific research will always remain an integral and indispensable part of the biological sciences, a ceiling is eventually reached by these approaches which can only be breached by holistic interdisciplinary techniques that integrate biological information into knowledge-based models of complex systems. Systems biology has emerged as the scientific challenge of present times, and looks set to hold this title for a few more years to come. In order to be able to benefit from current and future scientific developments, wine science has to embrace the 'omic' era and incorporate its toolbox of profiling technologies into standard research practices. A holistic approach towards understanding the complex metabolic and regulatory phenomena that characterize living systems will undoubtedly reveal many of the unknown interactions between genes, proteins, and metabolites, facilitating the truly rational improvement of production processes and the development of new biotechnological approaches to address current limitations of vine growing and wine making.

\section{LITERATURE CITED}

Adahchour, M., Beens, J., Vreuls, R.J. \& Brinkman U.A. 2006. Recent developments in comprehensive two-dimensional gas chromatography (GCxGC) IV. Further applications, conclusions and perspectives. Trends Anal. Chem. 25, 821-840.

Adams, J., Puskas-Rozsa, S., Simlar, J. \& Wilke, C.M., 1992. Adaptation and major chromosomal changes in population of Saccharomyces cerevisiae. Curr. Biol. 22, 13-19.

Allen J., Davey, H.M., Broadhurst, D., Heald, J.K., Rowland, J.J., Oliver, S.G. \& Kell, D.B., 2003. High-throughput classification of yeast mutants for functional genomics using metabolic footprinting. Nat. Biotechnol. 21, 692-696.

Anderson, S., 1981. Shotgun DNA sequencing using cloned DNase 1-generated fragments. Nucl. Acids Res. 9, 3015-3027.

Attfield, P.V., 1997. Stress tolerance: the key to effective strains of baker's yeast. Nat. Biotechnol. 15, 1351-1357.

Bailey, J., 1991. Towards a science of metabolic engineering. Science 252, 16681675 .

Bakalinsky, A.T. \& Snow, R., 1990. The chromosomal constitution of wine strains of Saccharomyces cerevisiae. Yeast 6, 367-382.

Beltran, G., Novo, M., Leberre, V., Sokol, S., Labourdette, D., Guillamon, J-M., Mas, A., François, J. \& Rozes, N., 2006. Integration of transcriptomic and metabolic analyses for understanding the global response of low-temperature winemaking fermentations. FEMS Yeast Res. 6, 1167-1183.

Boer, V.M., de Winde, J.H., Pronk, J.T. \& Piper, M.D., 2003. The genome-wide transcriptional responses of Saccharomyces cerevisiae grown on glucose in aerobic chemostat cultures limited for carbon, nitrogen, phosphorus or sulfur. J. Biol. Chem. 278, 3265-3274.

Borneman, A.R., Forgan, A., Pretorius, I.S. \& Chambers, P.J., 2008. Comparative genome analysis of a Saccharomyces cerevisiae wine strain. FEMS Yeast Res. 8, $1185-1195$

Brejning, J., Arneborg, N. \& Jespersen, L., 2005. Identification of genes and proteins induced during the lag and early exponential phase of lager brewing yeasts. J. Appl. Microbiol. 98, 261-271.

Brown, P.O. \& Botstein, D., 1999. Exploring the new world of the genome with microarrays. Nat. Genet. 21, 33-37.

Bruggeman, F.J. \& Westerhoff, H.V., 2007. The nature of systems biology. Trends Microbiol. 15, 45-50.

Çakır, T., Patil, K.R., Önsan, Z.I., Ülgen, K.O., Kirdar, B. \& Nielsen, J., 2006. Integration of metabolome data with metabolic networks reveals reporter reactions. Mol. Systems Biol. 2, 50.

Cambon, B., Monteil, V., Remize, F., Camarasa, C. \& Dequin, S., 2006. Effects of GPD1 overexpression in Saccharomyces cerevisiae commercial wine yeast strains lacking ALD6 genes. Appl. Environ. Microbiol. 72, 4688-4694.

Campo, E., Ferreira, V., Lopez, R., Escudero, A. \& Cacho, J., 2006. Identification of three novel compounds in wine by means of a laboratory-constructed multidimensional gas chromatographic system. J. Chrom. A. 1122, 202-208.

Cereghino, G.P. \& Cregg, J.M., 1999. Applications of yeast in biotechnology: protein production and genetic analysis. Curr. Opin. Biotechnol. 10, 422-427.

Chapman, J.W., 1991. The development and use of novel yeast strains for food and drink manufacture. Trends Food Sci. Technol. 2, 176-180.

Christensen, B. \& Nielsen, J., 1999. Isotopomer analysis using GC-MS. Metab. Eng. 1, 282-290. 
Cramer, G.R., Ergül, A., Grimplet, J., Tillett, R.L., Tattersall, E.A., Bohlman, M.C., Vincent, D., Sonderegger, J., Evans, J., Osborne, C., Quilici, D., Schlauch, K.A., Schooley, D.A. \& Cushman, J.C., 2007. Water and salinity stress in grapevines: early and late changes in transcript and metabolite profiles. Funct. Integr. Genomics 7, 111-134.

Daran-Lapujade, P., Jansen, M.L., Daran, J.M., Van Gulik, W., De Winde, J.H. \& Pronk, J.T., 2004. Role of transcriptional regulation in controlling fluxes in central carbon metabolism of Saccharomyces cerevisiae. A chemostat culture study. J. Biol. Chem. 279, 9125-9138.

Da Silva, F.G., Iandolino, A., Al-Kayal, F., Bohlmann, M.C., Cushman, M.A., Lim, H., Ergul, A., Figueroa, R., Kabuloglu, E.K., Osborne, C., Rowe, J., Tattersall, E., Leslie, A., Xu, J., Baek, J., DeRisi, J.L., Iyer, V.R. \& Brown, P.O., 1997. Exploring the metabolic and genetic control of gene expression on a genomic scale. Science $278,680-686$

De Jong, H., 2002. Modeling and simulation of genetic regulatory systems: a literature review. J. Comput. Biol. 9, 69-105.

De Jong, H., Geiselmann, J., Hernandez, C. \& Page, M., 2003. Genetic Network Analyzer: Qualtitative simulation of genetic regulatory networks. Bioinformatics 19, 336-344.

Dettmer, K., Aronov, P.A. \& Hammock, B.D., 2006. Mass spectrometry-based metabolomics. Mass Spectrom. Revs 26, 51-78.

Driesel, A., Lommele, A., Drescher, B., Topfer, R., Bell, M., Cartharius, I., Cheutin, N., Huck J-F., Kubiak, J., \& Regnard, P., 2003. Towards the transcriptome of grapevine (Vitis vinifera L.) Acta Hort. 603, 239-250.

Duarte, N.C., Herrgård, M.J. \& Palsson, B.O., 2004. Reconstruction and validation of Saccharomyces cerevisiae iND750, a fully compartmentalized genome-scale metabolic model. Genome Res. 14, 1298-1309.

Dunn, W.B. \& Ellis, D., 2005. Metabolomics: Current analytical platforms and methodologies. Trends Anal. Chem. 24, 285-294.

Edwards, J.S. \& Palsson, B.O., 2000. The Escherichia coli MG1655 in silico metabolic genotype: Its definition, characteristics, and capabilities. Proc. Natl. Acad. Sci. USA 97, 5528-5533.

Edwards, J.S., Ibarra, R.U. \& Palsson, B.O., 2001. In silico predictions of Escherichia coli metabolic capabilities are consistent with experimental data. Nat. Biotechnol. 19, 125-130.

Erasmus, D.J., Van der Merwe, G.K. \& Van Vuuren, H.J.J., 2003. Genome-wide expression analyses: Metabolic adaptation of Saccharomyces cerevisiae to high sugar stress. FEMS Yeast Res. 3, 375-399.

Famili, I., Forster, J., Nielsen, J. \& Palsson, B.O., 2003. Saccharomyces cerevisiae phenotypes can be predicted by using constraint-based analysis of a genome-scale reconstructed metabolic network. Proc. Natl. Acad. Sci. USA 100, 13134-13139. Farmer, W.R. \& Liao, J.C., 2000. Improving lycopene production in Escherichia coli by engineering metabolic control. Nat. Biotechnol. 18, 533-537.

Fey, S.J. \& Larsen, P.M., 2001. 2D or not 2D. Two-dimensional gel electrophoresis. Curr Opin. Chem. Biol. 5, 26-33.

Forster, J., Famili, I., Fu, P., Palsson, B.O. \& Nielsen, J., 2003. Genome-scale reconstruction of the Saccharomyces cerevisiae metabolic network. Genome Res. $13,244-253$

Forster, J., Famili, I., Palsson, B.O. \& Nielsen, J., 2003. Large-scale evaluation of in silico gene deletions in Saccharomyces cerevisiae. OMICS 7, 193-202.

Frezier, V. \& Dubourdieu, D., 1992. Ecology of yeast strain Saccharomyces cerevisiae during spontaneous fermentation in a Bordeaux winery. Am. J. Enol. Vitic. 43, 375-380.

Gasch, A.P., Spellman, P.T., Kao, C.M., Carmel-Harel, O., Eisen, M.B., Storz, G., Botstein, D. \& Brown, P.O., 2000. Genomic expression changes in the response of yeast cells to environmental changes. Mol. Biol. Cell 11, 4241-4257.

Gasch, A.P. \& Werner-Washburne, M., 2002. The genomics of yeast responses to environmental stress and starvation. Funct. Integr. Genomics 2, 181-192.

Ge, H., Liu, Z., Church, G.M. \& Vidal, M., 2001. Correlation between trancriptome and interactome mapping data from Saccharomyces cerevisiae. Nat. Genet. 29, 482-486.

Ge, H., Walhout, A.J. \& Vidal, M., 2003. Integrating 'omic' information: a bridge between genomics and systems biology. Trends Genet. 19, 551-560.

Goffeau, A., Barrell, B.G., Bussey, H., Davis, R.W., Dujon, B., Feldmann, H., Galibert, F., Hoheisel, J.D., Jacq, C., Johnston, M., Louis, E.J., Mewes, H.W., Murakami, Y., Philippsen, P., Tettelin, H. \& Oliver, S.G., 1996. Life with 6000 genes. Science $274,563-567$
Gombert, A.K., dos Santos, M.M., Christensen, B. \& Nielsen, J., 2001. Network identification and flux quantification in the central metabolism of Saccharomyces cerevisiae under different conditions of glucose repression. J. Bacteriol. 183, 1441-1451.

Goryanin, I., Hodgman, T.C. \& Selkov, E., 1999. Bioinformatics 15, 749758. Gregoriev, A., 2001. A relationship between gene expression and protein interactions on proteome scale: analysis of the bacteriophage T7 and the yeast Saccharomyces cerevisiae. Nucl. Acids Res. 29, 3513-3519.

Griffin, T.J., Gygi, S.P., Ideker, T., Rist, B., Eng, J., Hood, L. \& Aebersold, R., 2002. Complementary profiling of gene expression at the transcriptome and proteome levels in Saccharomyces cerevisiae. Mol. Cell Proteomics 1, 323-333. Hartwell, L.H., Hopfield, J.J., Leibler, S. \& Murray, A.W., 1999. From molecular to modular cell biology. Nature 402, C47-C52.

Heux, S., Sablayrolles, J.M., Cachon, R. \& Dequin, S., 2006. Engineering a Saccharomyces cerevisiae wine yeast that exhibits reduced ethanol production during fermentation under controlled microoxygenation conditions. Appl. Environ. Microbiol. 72, 5822-5828.

Hu, C.D. \& Kerppola, T.K., 2003. Simultaneous visualization of multiple protein interactions in living cells using multicolor fluorescence complementation analysis. Nat. Biotechnol. 21, 539-545.

Ideker, T., Galitski, T. \& Hood, L., 2001. A new approach to decoding life: systems biology. Annu. Rev. Genomics Hum. Genet. 2, 343-372.

Ideker, T., Thorsson, V., Ranish, J.A., Christmas, R., Christmas, R., Buhler, J., Eng, J.K., Bumgarner, R., Goodlett, D.R., Aebersold, R. \& Hood, L, 2001. Integrated genomic and proteomic analyses of a systematically perturbed metabolic network. Science 292, 929-934

Ideker, T. \& Lauffenberger, D., 2003. Building with a scaffold: emerging strategies for high- to low-level cellular modeling. Trends Biotechnol. 21, 255-262.

Ito, T., Chiba, T. \& Yoshida, M., 2001. Exploring the protein interactome using comprehensive two-hybrid projects. Trends Biotechnol. 19, S23-S27.

Jeong, H., Tombor, B., Albert, R., Oltvai, Z.N. \& A-L, Barabasi, 2000. The largescale organization of metabolic networks. Nature 407, 651-654.

Kolkman, A., Daran-Lapujade, P., Fullaondo, A., Olsthoorn, M.M.A., Pronk, J.T., Slijper, M. \& Heck, A.J.R., 2006. Proteome analysis if yeast response to various nutrient limitations. Mol. Sys. Biol. 2, 2006.0026.

Kuhn, K.M., DeRisi, J.L., Brown, P.O. \& Sarnow, P., 2001. Global and specific translational regulation in the genomic response of Saccharomyces cerevisiae to a rapid transfer from a fermentable to a nonfermentable carbon source. Mol. Cell. Biol. 21, 916-927.

Kresnowati, M.T., van Winden, W.A., Almering, M.J., Ten Pierick, A., Ras, C., Knijnenburg, T.A., Daran-Lapujade, P., Pronk, J.T., Heijnen, J.J. \& Daran, J.M., 2006. When transcriptome meets metabolome: fast cellular responses of yeast to sudden relief of glucose limitation. Mol. Syst. Biol. 2, 49.

Lambrechts, M.G. \& Pretorius, I.S., 2000. Yeast and its importance to wine aroma. S. Afr. J. Enol. Vitic. 21, 97-129.

Lander, E.S., 1999. Array of hope. Nat. Genet. 21, 3-4

Larsson, O., Wennmalm, K. \& Sandberg, R., 2006. Comparative microarray analysis. OMICS 10, 381-397.

Lavine, B. \& Workman, J., 2006. Chemometrics. Anal. Chem. 78, 4137-4145.

Lilly, M., Bauer, F.F., Styger, G., Lambrechts, M.G. \& Pretorius, I.S., 2006. The effect of increased branched-chain amino acid transaminase activity in yeast on the production of higher alcohols and on the flavour profiles of wine and distillates. FEMS Yeast Res. 6, 726-743.

Louw, C., La Grange, D., Pretorius, I.S. \& Van Rensburg, P., 2006. The effect of polysaccharide-degrading wine yeast transformants on the effciency of wine processing and wine flavour. Biotechnol. 125, 447-461.

Mack, G.S., 2004. Can complexity be commercialized? Nat. Biotechnol. 22, 1223-1229.

Malherbe, D.F., Du Toit, M., Cordero Otero, R.R., Van Rensburg, P. \& Pretorius, I.S., 2003. Expression of the Aspergillus niger glucose oxidase gene in Saccharomyces cerevisiae and its potential applications in wine production. Appl. Microbiol. Biotechnol. 61, 502-551.

Mann, M., Hendricksen, R.C. \& Pandey, A., 2001. Analysis of proteins and proteomes by mass spectrometry. Annu. Rev. Biochem. 70, 437-473. 
Marks, V.D., Ho Sui, S.J., Erasmus, D., van den Merwe, G.K., Brumm, J., Wasserman, W.W., Bryan, J. \& van Vuuren, H.J.J., 2008. Dynamics of the yeast transcriptome during wine fermentation reveals a novel fermentation stress response. FEMS Yeast Res. 8, 35-52.

Masneuf, I., Hansen, J., Groth, C., Piskur, J. \& Dubourdieu, D., 1998. New hybrids between Saccharomyces sensu stricto yeast species found among wine and cider production strains. Appl. Environ. Microbiol. 64, 3887-3892.

Mendes-Ferreira, A., del Olmo, M., Garcia-Martinez, J., Jimenez-Marti E, Mendes-Faia A., Perez-Ortin, J.E \& Leao, C., 2007. Transcriptional response of Saccharomyces cerevisiae to different nitrogen concentrations during alcoholic fermentation. Appl. Environ. Microbiol. 73, 3049-3060.

Nägele, E., Vollmer, M. \& Hörth, P., 2004. Improved 2D nano-LC/MS for proteomics applications: A comparative analysis using yeast proteome. J. Biomol. Techn. 15, 134-143.

Nielsen, J., 2003. It's all about metabolic fluxes. J. Bacteriol. 185, 7031-7035. Nielsen, J. \& Jewett, M.C., 2008. Impact of systems biology on metabolic engineering of Saccharomyces cerevisiae. FEMS Yeast Res. 8, 122-131.

O'Farrell, P.H., 1997. High resolution two-dimensional electrophoresis of proteins. J. Biol. Chem. 250, 4007-4021.

Palsson, B.O., 2000. The challenges of in silico biology. Nat. Biotechnol. 18, 1147-1150.

Palsson, B.O., 2002. In silico biology through 'omics'. Nat. Biotechnol. 20, 649-650.

Patil, K.R. \& Nielsen J, 2005. Uncovering transcriptional regulation of metabolism by using metabolic network topology. PNAS 102, 2685-2689.

Peng, J., Elias, J.E., Thoreen, C.C., Licklider, L.J. \& Gygi, S.P., 2002. Evaluation of multidimensional chromatography coupled with tandem mass spectrometry (LC/LC-MS/MS) for large-scale protein analysis: The yeast proteome. J. Proteome Res. 2, 43-50.

Pereira-Leal, J.B., Enright, A.J. \& Ouzounis, C.A., 2004. Detection of functional modules from protein interaction networks. Proteins 54, 49-57.

Pizarro, F.J., Jewett, M.C., Nielsen, J. \& Agosin, E., 2008. Growth temperature exerts differential physiological and transcriptional responses in laboratory and wine strains of Saccharomyces cerevisiae. Appl. Environ. Microbiol. 74, 63556368 .

Pretorius, I.S. \& Bauer, F.F., 2002. Meeting the consumer challenge through genetically customised wine yeast strains. Trends Biotechnol. 20, 426-432. Price, N.D., Papin, J.A., Schilling, C.H. \& Palsson, B., 2003. Trends Biotechnol. 21, 162-169.

Puig, S., Ramón, D., Pérez-Ortin, J.E., 1998. Optimized method to obtain stable food-safe recombinant wine yeast strains. J. Agric. Food Chem. 46, 1689-1693.

Puig, S. \& Pérez-Ortin, J.E., 2000. Stress response and expression patterns in wine fermentation of yeast genes induced at the diaxic shift. Yeast 16, 139-148.

Rabilloud, T., 2002. Two-dimensional gel electrophoresis in proteomics: old, old fashioned, but it still climbs the mountains. Proteomics 2, 3-10.

Rachidi, N., Barre, P. \& Blondin, B., 1999. Multiple Ty-mediated chromosomal translocation lead to karyotype changes in a wine strain of Saccharomyces cerevisiae. Mol. Gen. Genet. 261, 841-850.

Reed, J.L \& Palsson, B.O., 2003. Thirteen years of building constraint-based in silico models of Escherichia coli. J. Bacteriol. 185, 2692-2699.

Ronaghi, M., Uhlén, M. \& Nyrén, P., 1998. A sequencing method based on realtime pyrophosphate. Science $281,363,365$.

Rossignol, T., Dulau, L., Julien, A. \& Blondin, B., 2003. Genome-wide monitoring of wine yeast gene expression during alcoholic fermentation. Yeast 20, 1369-1385.

Rossouw, D., Naes, T. \& Bauer, F.F., 2008. Linking gene regulation and the exometabolome: A comparative transcriptomics approach to identify genes that impact on the production of volatile aroma compounds in yeast. BMC Genomics 9, 530 .

Salvadó, Z., Chiva, R., Rodriguez-Vargas, S., Rández-Gil, F., Mas, A. \& Guillamón, J.M., 2008. Proteomic evolution of a wine yeast during the first hours of fermentation. FEMS Yeast Res. 8, 1137-1146.

Sauer, U., 2004. High-throughput phenomics: experimental methods for mapping fluxomes. Curr. Opin. Biotechnol. 15, 58-63.

Smolka, M., Zhou, H. \& Aebersold, R., 2002. Quantitative protein profiling using two-dimensional gel electrophoresis, isotope-coded affinity tag labeling, and mass spectrometry. Mol. Cell. Proteomics 1, 19-29.
Stephanpoulos, G., 1999. Metabolic fluxes and metabolic engineering. Metab. Eng. 1, 1-10.

Stephanopoulos, G., Alper, H., Moxley J.F., 2004. Exploiting biological complexity for strain improvement through systems biology. Nat. Biotechnol. 22, 1261-1267.

Smedsgaard, J. \& Nielsen, J., 2005, Metabolite profiling of fungi and yeast: from phenotype to metabolome by MS and informatics. J. Exp. Bot. 56, 273-286.

Szyperski, T., 1998. 13C-NMR, MS and metabolic flux balancing in biotechnology research. Q. Rev. Biophys. 31, 41-106.

Templin, M.F., Stoll, D., Bachmann, J. \& Joos T.O., 2004. Protein microarrays and multiplexed sandwich immunoassays: what beats the beads? Comb. Chem. High Throughput Screen. 7, 223-229.

Tong, A.H., Drees, B., Nardelli, G., Bader, G.D., Brannetti, B., Castagnoli, L., Evangelista, M., Ferracuti, S., Nelson, B., Paoluzi, S., Quondam, M., Zucconi, A., Hoque, C.W., Fields, S., Boone, C. \& Cesareni, G., 2002. A combined experimental and computational strategy to define protein interaction networks for peptide recognition modules. Science 295, 321-324.

Uetz, P., Giot, L., Cagney, G., Mansfield, T.A., Judson, R.S., Knight, J.R., Lockshon, D., Narayan, V., Srinivasan, M., Pochart, P., Qureshi-Emili, A., Li, Y., Godwin, B., Conover, D., Kalbfleisch, T., Vijayadamodar, G., Yang, M., Johnson, M., Fields, S. \& Rothberg, J.M., 2000. A comprehensive analysis of proteinprotein interactions in Saccharomyces cerevisiae. Nature 403, 623-627.

Usaite, R., Patil, K.R., Grotkjær, T., Nielsen, J. \& Regenberg, B., 2006. Global transcriptional and physiological responses of Saccharomyces cerevisiae to Ammonium, L-Alanine, or L-Glutamine limitation. Appl. Environ. Microbiol. 72, 6194-6203.

Varela, C., Cardenas, J., Melo, F. \& Agosin, E., 2005. Quantitative analysis of wine yeast gene expression profiles under winemaking conditions. Yeast 22, 369-383.

Vemuri, G.N. \& Aristidou, A.A., 2005. Metabolic engineering in the -omics era: Elucidating and modulating regulatory networks. Microbiol. Mol. Biol. Revs 69, 197-216.

Vido, K., Spector, D., Lagniel, G., Lopez, S., Toledano, M.B. \& Labarre, J., 2001. A proteome analysis of the Cadmium response in Saccharomyces cerevisiae. J. Biol. Chem. 276, 8469-8474.

Villas-Bôas, S.G., Mas, S., Åkesson, M., Smedsgaard, J. \& Nielsen, J., 2005. Mass spectrometry in metabolome analysis. Mass Spectrom. Revs 24, 613-646.

Villas-Bôas, S.G., Roessner, U., Hansen, M.A.E., Smedsgaard, J. \& Nielsen, J., 2007. Yeast Metabolomics: The discovery of new metabolic pathways in Saccharomyces cerevisiae. In: Metabolome Analysis. John Wiley \& Sons, Inc.

Volschenk, H., Viljoen, M., Grobler, J., Petzold, B., Bauer, F., Subden, R.E., Young, R.A., Lonvaud-Funel, A., Denayrolles, M. \& Van Vuuren, H.J.J., 1997. Engineering pathways for malate degradation in Saccharomyces cerevisiae. Nat. Biotechnol. 15, 253-257.

Volschenk, H., Viljoen, M., Grobler, J., Bauer, F., Lonvaud-Funel, A., Denayrolles, M., Subden, R.E. \& Van Vuuren, H.J.J., 1997. Malolactic fermentation in grape musts by a genetically engineered strain of Saccharomyces cerevisiae. Am. J. Enol. Vitic. 48, 193-197.

Walhout, A.J., Reboul, J., Shtanko, O., Bertin, N., Vaglio, P., Ge, H., Lee, H., Doucette-Stamm, L., Gunsalus, K.C., Schetter, A.J., Morton, D.G., Kemphues, K.J., Reinke, V., Kim, S.K., Piano, F. \& Vidal, M., 2002. Integrating interactome, phenome, and transcriptome mapping data for the C. elegans germline. Curr. Biol. 12, 1952-1958.

Walter, G., Bussow, K., Cahill, D., Lueking, A. \& Lehrach, H., 2000. Protein arrays for gene expression and molecular interaction screening. Curr. Opin. Microbiol. 3, 298-302.

Washburn, M.P., Ulaszek, R., Deciu, C., Schielts, D.M. \& Yates, J.R., 2002. Analysis of quantitative proteomic data generated via multidimensional protein identification technology. Anal. Chem. 74, 1650-1657.

Weiner, H., Faupel, T. \& Bussow, K., 2004. Protein arrays from cDNA expression libraries. Methods Mol. Biol. 264, 1-13.

Wiechert, W., Siefke, C., de Graaf, A.A. \& Marx, A., 1997. Bidirectional reaction steps in metabolic networks. II. Flux estimation and statistical analysis. Biotechnol. Bioeng. 55, 118-135.

Wiechert, W., 2001. 13C metabolic flux analysis. Metab. Eng. 3, 195-206.

Zhu, H. \& Snyder, M., 2003. Protein chip technology. Curr. Opin. Chem. Biol. $7,55-63$. 